\title{
Detection of early lung cancer
}

\author{
Joanna C Porter, Stephen G Spiro \\ Department of Thoracic Medicine, The Middlesex Hospital, London W1N 8AA, UK
}

Introductory article

\section{Early Lung Cancer Action Project: overall design and findings from baseline screening}

\author{
CI Henschke, DI McCauley, DF Yankelevitz, DP Naidich, G McGuinness, OS Miettinen, DM Libby, \\ MW Pasmantier, J Koizumi, NK Altorki, JP Smith
}

\begin{abstract}
Background. The Early Lung Cancer Action Project (ELCAP) is designed to evaluate baseline and annual $\overrightarrow{\text { if }}$ repeat screening by low-radiation-dose computed tomography (low-dose CT) in people at high risk of S $^{2}$ lung cancer. We report the baseline experience. Methods. ELCAP has enrolled 1000 symptom-free $\bigcirc$ volunteers, aged 60 years or older, with at least 10 pack-years of cigarette smoking and no previous $\vec{\longrightarrow}$ cancer, who were medically fit to undergo thoracic surgery. After a structured interview and informed consent, chest radiographs and low-dose CT were done for each participant. The diagnostic investigation of screen-detected non-calcified pulmonary nodules was guided by ELCAP recommendations, which $\tilde{\circ}$ included short-term high-resolution CT follow-up for the smallest non-calcified nodules. Findings. Non-. calcified nodules were detected in 233 (23\% [95\% Cl 21-26]) participants by low-dose CT at baseline, 8 compared with 68 (7\% [5-9]) by chest radiography. Malignant disease was detected in $27(2.7 \%$ [1.8-3.8]) by CT and seven (0.7\% [0.3-1.3]) by chest radiography, and stage I malignant disease in 23 o (2.3\% [1.5-3.3]) and four (0.4\% [0.1-0.9]), respectively. Of the 27 CT-detected cancers, 26 were resectable. Biopsies were done on 28 of the 233 participants with non-calcified nodules; 27 had malignant noncalcified nodules and one had a benign nodule. Another three individuals underwent biopsy against the ELCAP recommendations; all had benign non-calcified nodules. No participant had thoracotomy for a benign nodule. Interpretation. Low-dose CT can greatly improve the likelihood of detection of small non-calcified nodules, and thus of lung cancer at an earlier and potentially more curable stage. $\vec{F}$ Although false-positive CT results are common, they can be managed with little use of invasive diagnostic procedures. (Lancet 1999;354:99-105)
\end{abstract}

Lung cancer is common, fatal if untreated, and responsible each year for more deaths in the USA and Western Europe than colorectal, cervical, and breast cancer combined. Those most at risk are current smokers, but rates of lung cancer remain high many years after smoking cessation ${ }^{2}$ with estimates of 92 million ex-smokers at risk of the disease in the USA alone. This means that, in the unlikely event that a combination of education and public health policies make smoking a sin of the past, the scourge of lung cancer will still be with us for the foreseeable future. Despite the high incidence of lung cancer, up to date treatments have had no worthwhile impact on the gloomy five year survival figure of $7-13 \% 3^{3}$ which has remained unchanged for 30 years. Cure rates are highly dependent on tumour staging. If patients present early with resectable stage I disease, five year survival can be as high as $70 \% 7_{11}^{11}$ However, the presenting symptoms of lung cancer occur late in the natural history of the illness, with up to $80 \%$ of patients having unresectable advanced disease and only $20 \%$ of lung cancers being picked up as stage I growths. ${ }^{3}$ To improve lung cancer mortality we need to intercept more patients much earlier, ideally with stage I disease, from an asymptomatic at risk population. The screening tools of chest $\stackrel{\sim}{\sigma}$ radiography and sputum cytology are safe, effective, $N$ cheap, and available and yet - in contrast to colorectal, N cervical, and breast cancer - there are currently no $\underset{0}{0}$ screening programmes for early detection of stage I lung cancer. Why not? Four randomised control trials (RCTs) screening male smokers in the 1970 s all failed to show $\stackrel{\mathbb{\Phi}}{\rightarrow}$ a reduction in mortality from lung cancer ${ }^{12}-19$ and the nihilistic mantra that screening for lung cancer does not

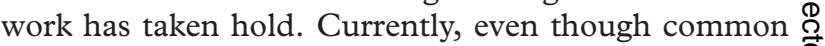
sense and clinical experience argue that early detection $\stackrel{\mathbb{Q}}{\varrho}$ and treatment of lung cancer is advantageous, no advisory committee recommends mass screening of at risk 8 patients. Three decades on, it is remarkable that these RCTs, with their own shortcomings, have been so 흘 powerful in dismissing the potential of screening. It is refreshing, timely, and appropriate that recent interest in the use of helical computerised tomographic (CT) scanning and biomedical markers of early malignant change have rekindled an interest in screening for lung 
cancer and a review of the prevailing dogma that screening does not save lives. The Early Lung Cancer Açtion Project (ELCAP) study (the introductory article 20 and a recent Japanese study that also used CT screenin ${ }^{2}$ aim to establish a curability rate based on the size of lung cancers detected by screening. The hope is that, if the percentage of lung cancers detected at stage I can be increased from the current figure of $20 \%$, mortality advantages will follow. If so, then an RCT to examine whether widespread screening for lung cancer is justified would be an urgent priority in the fight against this disease.

The past - the 1970s: paving the way for nihilism In the 1970 s, with increasing rates of lung cancer in the USA, the National Cancer Institute (NCI) sponsored three large RCTs in the context of the Cooperative Early Lung Detection Program; a fourth RCT took place in Czechoslovakia.

\section{THE MAYO LUNG PROJECT}

The Mayo Lung Project $\left.\right|^{1}||^{1} \mid 18$ compared regular screening with infrequent, sporadic, or no screening in a control group. The participants were men aged 45 or older who had smoked 20 cigarettes or more a day for the year before entry to the study. Prevalent cases of lung cancer were excluded by chest radiography and three day pooled sputum cytological examination. The screening group underwent chest radiography and sputum cytology every four months for six years whilst the control group were given the "standard Mayo advice" of the time which was to have an annual chest radiograph and sputum cytological examination. Patients were not reminded of this advice. The experimental and follow up period lasted for an average of nine years in total. At the conclusion of the study 160 of 4595 cancers were detected in the control group (none through selfreferral for screening chest radiographs) and 206 of 4618 in the screened group ( $44 \%$ of which were picked up during screening studies rather than as a result of symptoms), giving a cumulative incidence of lung cancer in experimental and control populations of $4.5 \%$ and $3.5 \%$, respectively. The number of deaths from lung cancer, the death rate from lung cancer per 1000 person years, and the all-cause mortality were unaffected by screening. This led to the conclusion that differences between the two groups could be explained by lead time bias, length biased sampling, and/or overdiagnosis. The paradox that a favourable shift in stage distribution did not translate into an improvement in lung cancer mortality remained unexplained.

THE CZECHOSLOVAKIAN LUNG CANCER SCREENING STUDY The Czechoslovakian lung cancer screening study enrolled male smokers aged 40-64 years with a life time cigarette consumption of approximately 20.5 pack years. ${ }^{1}$ Prevalent cases were excluded by chest radiography and 24 hour sputum cytological examination. The screened group had chest radiographs and sputum cytological screening every six months for three years whilst the controls had a single screen at the end of the study. Both groups then had chest radiographs annually for three further years. At the end of the three year study period, before the final three year radiograph, 36 lung tumours had been identified in the study group (75\% of which were picked up through screening) and 19 in the control group. Resectability was $25 \%$ and
$15 \%$ in the screened and control groups, respectively. Just as in the Mayo project, five year survival in the patients diagnosed with lung cancer was significantly better in the screened group $(23 \%$ versus $0 \%, \mathrm{p}=$ $0.0001)$. However, lung cancer mortality was higher in $x$ the screened group after three years (28 versus $18 \overrightarrow{\overrightarrow{\mathrm{N}}}$ deaths). After six years there were 108 cases of lung $\overrightarrow{0}$ cancer (85 deaths) in the screened group and 82 lung $\frac{E}{0}$ cancers (67 deaths) in the control group, an insignificant $\frac{\bar{m}}{\mathrm{~m}}$ difference in lung cancer mortality $(\mathrm{p}=0.16)$. Again, as $\mathbb{\nabla}$ for the Mayo Lung Project, although there were benefits in stage distribution, curability and disease specific fatality in the screened group, these did not translate into $\vec{\circ}$ decreased mortality from lung cancer, the only end $\overrightarrow{\vec{\omega}}$ point deemed free from bias.

DUAL VERSUS SINGLE SCREENIN

Johns Hopkins Hespita ${ }^{13}$ and Memorial-Sloan- G Kettering Hospita ${ }^{1}{ }^{22}$ both randomised male smokers 穴 over the age of 45 to undergo annual chest radiographic 음 examination and three day pooled sputum cytology $\rightarrow$ every four months while a control group underwent of only annual chest radiography. Neither hospital showed o any benefit to lung cancer mortality from the addition 윽 of sputum cytological examination. Less than $10 \%$ of $\vec{x}$ cancers were detectable by sputum cytology alone which might reflect the poor sensitivity of sputum cell morphological studies that were available at the time. In- $\vec{N}$ terestingly, the stage distributions and five year survival of all men who developed lung cancer were more fa- . vourable than the national average (35\% versus $13 \%) .8$

CRITICISM OF THE EARLY STUDIES

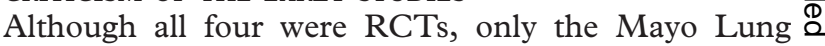
Project had a true control group that was unscreened. $\overrightarrow{\overrightarrow{0}}$ The design of the Mayo trial was such that it lacked 3 power from the outset with less than $20 \%$ power to detect a $10 \%$ benefit in lung cancer mortality and 55\% power to detect a $20 \%$ benefit. This lack of power was $\overrightarrow{\vec{\partial}}$ increased even further by contamination of the control 을 group, of whom $55 \%$ had had a chest radiograph in the $\stackrel{x}{*}$ last year and $73 \%$ in the final two years. In addition, 3 compliance between the groups was low at $75 \%$ for the screened and $50 \%$ for the control group. As well as 3 being a weak study, the screening regime appeared 0 inexplicably ineffectual. Over the screening period only 45 of 206 lung cancers $(22 \%)$ picked up in the screened $\frac{D}{2}$ group were resectable compared with a $60 \%$ resectablility of the lung cancers picked up in both groups at $\sigma$ baseline. Given that so many problems and criticisms $N$ have been waged at this study, it is even more remarkable $N$ that public health policy has been so influenced by it.

INCREASED INCIDENCE OF LUNG CANCERS IN THE SCREENED GROUP

Of enormous interest and importance is the significant excess of diagnosed lung cancers in the screened group $\frac{\mathrm{Q}}{+}$ in the Mayo Lung Project ( $\mathrm{p}=0.016$; mainly early stage, $\stackrel{\mathrm{Q}}{\mathrm{Q}}$ resectable squamous cell carcinomas plus early stage and some advanced adenocarcinomas) which also ap- ᄋ్ proached significance in the Czechoslovakian study $(\mathrm{p}=$ 으 $0.065)$. Given that there is no evidence that the level of $\overline{\overline{0}}$ screening radiation was harmful, the excess must reflect $\stackrel{+}{?}$ a problem with study design such as misdiagnosis, overdiagnosis in the study group (finding lung cancers that would never be clinically important), underdiagnosis in the control group, or population hetero- 
geneity. Epidemiological data and the biological virulence of lung cancer argue that indolent lung cancers are vanishingly rare and overdiagnosis is therefore unlikely to explain the difference. Incorrect diagnosis is a better candidate; not all of the cancers were diagnosed by thoracotomy and necropsy and some labelled as primary lung cancers might have been, for example, metastatic adenocarcinomas. Underdiagnosis in the control group is certainly possible if participants died of competing disease before diagnosis of their lung cancer, and this is likely because of the high rates of coexistent cardiovascular disease in these populations. However, none of these diagnostic inaccuracies can explain the increase in "missing cases" in the follow up period of the Czechoslovakian study when control and screened groups were treated as one, and all underwent annual chest radiography. The only explanation for a continued difference in lung cancer between the two populations is that there were uncontrolled differences between them all along. Asbestos exposure 23 radon exposure ${ }^{24}$ chronic obstructive pulmonary disease, ${ }^{23}$ and genetic backgroun $\alpha^{2} \|^{27}$ all affect lung cancer rates in smokers, even after controlling for cigarette consumption. In reality, the excess lung cancer cases can be explained by invoking a combination of inaccurate diagnoses and population heterogeneity, and further question the design of these studies ${ }^{28}$

\section{POPULATION HETEROGENEITY AND DIAGNOSTIC \\ INACCURACY}

Disease specific mortality is the gold standard for evaluating the effects of screening within an RCT as it is the only end point not affected by lead time bias, length time bias, or overdiagnosis. In the context of an RCT, inaccurate or underdiagnosis does not affect the mortality end point but will affect the disease specific mortality. Population heterogeneity has a much more sinister effect. The RCT requires generation by randomisation of two equivalent groups with an equivalent risk of dying from a certain disease. If large enough, the groups will be comparable both for known and for unrecognised confounding variables. If randomisation produces two groups that differ in disease incidence, mortality comparisons between the two groups become impossible. Comparisons have even less meaning if the disease incidence between the heterogeneous groups is low. Compare the groups in a clinical trial in which everyone has the disease and are only a short time from diagnosis with those in a screening trial in which no one has the disease and only $1-5 \%$ will get it. A small difference of $1 \%$ in the total incidence in the Mayo Lung Project translates into a greatly increased proportional incidence of $20-40 \%$ in disease specific incidence. Small differences in disease incidence and mortality can therefore be grossly misinterpreted if the two groups are not adequately matched.

\section{The present - a new millennium: renewed enthusiasm}

A REAPPRAISAL OF CHEST RADIOGRAPHIC SCREENING

The Prostate, Lung, Colon and Ovarian (PLCO) Study is an RCT funded by the National Cancer Institute that aims to evaluate the role of screening in reducing mortality from these four common neoplasms. ${ }^{2} 3^{30}$ The study was set up to address some of the concerns raised over the design and interpretation of previous cancer screening trials and aims to recruit 148000 men and women aged $60-74$ years. They will be randomised into three groups on the basis of smoking history (current smoker, former smoker, and never smoked) to be screened or not screened. The screening for lung cancer will involve an annual chest radiograph. The study has an estimated power of 0.89 to detect a difference in $x$ lung cancer mortality of $10 \%$ and a power of 0.99 to $\overrightarrow{\overrightarrow{\vec{\omega}}}$ pick up a difference of $20 \%$. Although the design will $\overrightarrow{0}$ overcome some of the deficiencies of earlier studies, the $\frac{\bar{\sigma}}{\sigma}$ problems of population heterogeneity and confounding $\overline{\frac{\bar{m}}{\sigma}}$ risk factors may still not be adequately controlled.

HELICAL COMPUTED TOMOGRAPHY AS A SCREENING TOOL $\overrightarrow{0}$ Although there is renewed interest in chest radiographic $\overrightarrow{\vec{\omega}}$ screening, information since the Mayo trial suggests $\stackrel{\sim}{\mathscr{\omega}}$ that this is an insensitive tool. The chest radiograph is $\overrightarrow{\overrightarrow{5}}$ especially inadequate at picking up those lesions for which early detection is most beneficial - that is, lesions $\stackrel{\times}{0}$ of $2 \mathrm{~cm}$ in size, stage I adenocarcinomas, and rapidly or growing small cell and squamous cell carcinomas. ${ }^{31}$ ? Recognition of this fundamental limitation inspired the 음 search for other screening methods. Meanwhile, tech- nological advances produced the helical (or spiral) CT on scan with an estimated potential to detect $80-85 \%$ of lung cancers at stage I ${ }^{32}$ In the past CT scanning was disregarded for lung cancer screening because of $\vec{\longrightarrow}$ problems with availability, acceptability, and expense. Computed tomography delivers a high radiation dose $\frac{\vec{C}}{\omega}$ and the images produced require lengthy specialist in- $\vec{N}$ terpretation with a higher cost-benefit ratio than plain 8 chest radiography. This perception has altered dra-. matically with the introduction of fast, low dose (50 $\mathrm{mA}$ ), spiral CT scanners which produce a radiation dose one sixth that of a conventional CTscanner and only 10 times that of chest radiography. ${ }^{32}$ Screening

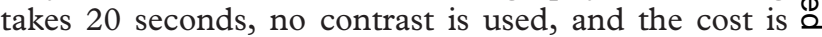
only slightly more than a chest radiograph $33_{3} 3$ The $\overrightarrow{\overrightarrow{0}}$ International Commission of Radiological Protection 3 estimates the nominal fatal cancer risk factor to be $5 \times$ F $10^{-5} / \mathrm{mSv}$ so that the estimated risk of causing a fatal cancer for an effective dose of $0.9-1.5 \mathrm{mSv}$ is calculated at about one in 26000 person years. An even newer development is the ultra low dose mobile scanner of $-6 \mathrm{~mA}$ (resolution falls off at $3 \mathrm{~mA}$ ) 34

The increasing availability of low dose spiral CT scanning at a time when trials for early lung cancer 3 detection were undergoing re-evaluation in the light of recognised limitations of trial design and screening methods paved the way for two key projects in the 1990s. Both of these studies were designed to evaluate the role of low dose spiral CT scanning in the early of detection of lung cancer. The study by Sone and col- $\mathrm{O}$ leagues in Matsumoto, Japan presented preliminary data స్ట from the first RCT of low dose spiral CT scanning as a mass screening tool ${ }^{21}$ and last year saw the publication 0 of the first report from the Early Lung Cancer Action $\bar{D}$ Project (ELCAP) in which Henschke and colleagues? presented their study design and results of baseline screening 20

THE MATSUMOTO STUDY

The first and only RCT to compare mobile CT scanning 8 and chest radiography in mass screening for early lung cancer was set up in 1996 to screen a Japanese popu- 흘 lation at low risk of the disease. ${ }^{21}$ A mobile, low dose, $\stackrel{?}{?}$ helical CT scanner (50 mA, without contrast) was used to screen unselected volunteers. These were smokers and non-smokers aged 40-74 years who had already undergone annual chest radiography (miniature fluoro- 
photography) and sputum cytology as part of a national screening programme. Of the participants, 3967 underwent both miniature fluorophotography and low dose helical chest CT scanning, and each was matched with two controls from the same population who underwent miniature fluorophotography only. Smokers from both groups underwent cytological examination of a 72 hour sputum collection. Each CT scan was read by one of four radiologists who classified the abnormalities. Participants with suspicious lesions $(59 / 3967,1 \%)$, indeterminate nodules $(80 / 3967,2 \%)$, and suspected cancer $(84 / 3967,2 \%)$ were followed up with conventional chest radiography, high resolution CT scanning, and transbronchial biopsy where possible. Of the 3967 participants, 223 underwent further examinations and 19 $(0.48 \%)$ were diagnosed with histologically confirmed lung cancer. In only one of the 19 patients was the abnormality seen on miniature fluorophotography and correctly interpreted, eight had abnormalities on chest radiography, and one other on a lateral view. High resolution CT scanning missed one central carcinoma that was picked up by sputum cytology. This gives an initial sensitivity for the protocol of 0.95 which will presumably decrease as missed cancers become apparent. Of the 19 cancers, 16 were American Joint Committee stage I and three were stage IV; 12 of the 19 were peripheral adenocarcinomas. Although large numbers of participants were screened and underwent further investigation, the pick up rate for cancer was relatively low giving a positive predictive value (PPV) of $8.5 \%$ (19/223). The lung cancer incidence of five per 1000 people screened was much greater than expected for the population $(\times 2.6$ for men and $\times 15.7$ for women) and, strikingly, there was no difference in lung cancer rates between smokers and non-smokers. Although the results may seem encouraging, it is worth bearing in mind that, to find 16 resectable cancers, 223 participants were examined with chest radiography, high resolution CT scanning, and some with transbronchial biopsy, 204 of whom did not have anything wrong. This has obvious implications in health care planning and economics. Mortality data comparing the screened population with their matched controls is not yet available but will be presented at the end of a one year follow up period.

\section{THE EARLY LUNG CANCER ACTION PROJECT (ELCAP)} ELCAP was initiated in 1992 as a non-comparative trial to allow fast and cost effective collection of information with two main aims. The first was to establish the role of annual low dose helical CT scanning in the diagnosis of early lung cancer by the evaluation of pulmonary nodules (as opposed to large central lesions). ${ }^{20}$ This involved the screening of high risk participants to document the incidence of pulmonary nodules as detected by CT scanning and is to be complemented by follow up data on rates of malignancy and resectability and, ultimately, on the relation between nodule size at detection and survival and cure. The second aim was to use this data base as a gold standard against which other studies of early lung cancer detection such as biomedical screening tools could be compared. The study recruited 1000 symptom free volunteers who were at a higher risk of lung cancer than in the Matsumoto study. The entry age was 60 years or over with a smoking history of at least 10 pack years (median 45 pack years), and subjects had to be deemed fit for thoracotomy. Each participant underwent chest radiography and low dose helical CT scanning ( $40 \mathrm{~mA})$. ELCAP laid down specific recommendations for the classification and further investigation of non-calcified nodules (NCNs) which included a short term follow up high resolution CT scan for the smallest NCNs. At the initial screening low dose CT scanning picked up NCNs in 233 participants (compared with 68 identified by chest radiography). All $\overrightarrow{\vec{*}}$ 233 then underwent conventional helical CT scanning $\overrightarrow{0}$ and biopsy samples were taken from 28 subjects, of $\frac{}{0}$ which 27 were malignant (PPV 27/233; 11.6\%). Of $\overline{\bar{p}}$ these, 18 were adenocarcinomas and there were no $\mathbb{8}$ small cell lung cancers. Stage I carcinomas made up 23 of the 27 tumours of which only four were visible on the chest radiograph and $26(96.3 \%)$ were resectable. This last figure compares dramatically with the re- $\overrightarrow{\vec{\omega}}$ sectability of only 30 of the 59 cancers that were picked $\stackrel{\omega}{\sigma}$ up in the Mayo Project at baseline chest radiographic $\overrightarrow{\overrightarrow{5}}$ screening. Malignant disease was also found by low dose CT scanning in four other participants - endobronchial or disease in two and mediastinal in the other two. These on were not included in the quantitative analysis as the $\frac{\text { ? }}{0}$ study was examining nodules. Again, the prevalence of malignant disease (31 per 1000) was higher than $\overrightarrow{-}$ expected. ISSUES OF SENSITIVITY AND SPECIFICITY
Both these studies ${ }^{20}$ raise issues of sensitivity and $\frac{\text { क }}{\text { क }}$ specificity. The number of malignancies picked up at initial screening in both studies is higher than expected, 8 with a striking preponderance of adenocarcinomas and . an absence of small cell carcinomas. This has raised the question of whether these peripheral adenocarcinomas, which are not easily seen on chest radiographs and may $\overline{\bar{o}}$ be very slow growing, are clinically relevant. In addition, 을

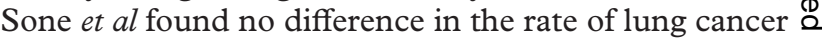
between smokers and non-smokers. These findings raise $\overrightarrow{\overrightarrow{0}}$ the possibility that small cell lung cancers detected by CT scanning have a different tumour biology from stage I lung cancers detected by chest radiography which may be altered further by smoking. Another possibility is $\overrightarrow{\overrightarrow{\hat{T}}}$ that the higher incidence of lung cancers in the Matsumoto study simply reflects those tumours missed by the previous year's annual screen. If this is the case, $\underline{3}$. then the incidence figures in subsequent years will return to expected levels and would strongly suggest that the $\frac{0}{3}$ tumours are all biologically relevant but, because they are picked up earlier, they are over-represented in the initial screen. This explanation would be supported if the ELCAP's one year follow up shows that a large proportion (for example, 30-40\%) of new nodules are $\Omega$ malignant, a finding that would increase the PPV of $\mathrm{N}$ subsequent screens from that of the original baseline $\mathrm{N}$ value. Given the lack of small cell carcinomas in the $\widetilde{C}$ initial screen despite an expected incidence of $20 \%{ }^{3}$ it will be interesting to see the cell types of these new nodules. These data, together with information on cost effectiveness, will be published later this year. Another worry, especially given the absence of certain cell types, is $\frac{O}{\mathbb{D}}$ the potential problem of underdiagnosis if lung nodules were incorrectly classified as benign and biopsy samples 음 were not taken. Any missed cancers will become apparent provided the follow up period is long enough. ᄋ̊ The one year follow up suggested for the study by Sone et $a l$ is too short and should be extended to allow proper data on sensitivity to be collected. It is hoped that these $\stackrel{?}{?}$ data will be provided by the ELCAP but may take some time. Also of concern is that $70 \%$ of all lung cancers arise in the large proximal airways and are visible at bronchoscopy, but the CT based studies have focused 
attention on the peripheral intrapulmonary lesions. Only regular follow up will provide data on the incidence and detection of the more common central tumours.

\section{BIOMEDICAL SCREENING TOOLS}

The morphology of epithelial cells found by cytological examination of sputum has been used since the 1930s for the diagnosis of early and advanced lung cancer and is most helpful for the detection of central tumours arising from the larger bronchi (usually squamous and small cell carcinomas). Further to the development of low dose CT scanners, there have been dramatic developments in the understanding of tumour biology and the development of biomedical markers for early premalignant change such as immunostaining of abnormal epithelial cells and polymerase chain reaction (PCR) based techniques to detect early genetic changes ${ }^{35}$ As such, these new techniques may complement low dose helical CT imaging which is less sensitive for detecting central tumours and premalignant changes. One cell surface marker is the heterogeneous nuclear ribonucleoprotein (hnRNP) A2/B1 which is upregulated on premalignant bronchial epithelial cells. In a pilot study of sputum archived from the Johns Hopkins screening study, overexpression of A2/B1 was a more sensitive marker of early preinvasive malignancy than normal cytological screening (sensitivity $91 \%$, specificity $88 \%$ ). In fact, features of malignancy were identifiable about one year before the conventional cytological examination showed abnormalities or the tumour was visible on the chest radiograph ${ }^{3 / 37}$ Similar encouraging results have been shown in prospective trials of Chinese tin miners ${ }^{38}$ North American lung cancer patients who have undergone resection of their primary tumour but are at high risk of recurrent disease 3 . and UK patients under investigation for lung cancer.4 Another approach has been to look at early chromosomal and genetic alterations in lung epithelial cells. In a retrospective study Mao et al found that point mutations in the p53 and K-ras genes in sputum samples preceded the clinical diagnosis of lung cancer in one case by more than one year ${ }^{41}$ Other groups have identified areas of genomic instability which cause microsatellite alterations that can act as clonal markers of early malignant disease. ${ }^{42}$

The limitations of sputum examination have led to the development of more invasive procedures to retrieve samples for biomedical analysis. Whilst fibreoptic bronchoscopy with white light may be used to detect preinvasive lung cancers, examination with fluorescent light greatly increases the detection of non-invasive cancers and precursor lesions ${ }^{45}$ Similarly, although the distal lung cannot be visualised bronchoscopically, the peripheral airways can be sampled using bronchoalveolar lavage (BAL). The predominant cells retrieved following $\mathrm{BAL}$ are alveolar macrophages and lymphocytes and the relative numbers of epithelial cells are small. These cells can then be subject to the same screening tests applied to expectorated sputum or lavage fluid. . $^{4}$ | 47 There is also evidence that microsatellite abnormalities can be detected in the plasma of patients with localised non-small cell lung carcinomas but not in controls, which gives the exciting prospect of using a blood test to screen for lung cancer ${ }^{48}$ However, although these tests are highly specific, their sensitivity is low and least helpful in patients with small, peripherally located, tumours and, as yet, are not appropriate for screening purposes. ${ }^{49}$

One further interesting observation with screening potential is the finding of volatile organic compounds (VOCs), particularly alkanes and benzene derivatives, in the breath of patients with lung cancer. A combination of 22 VOCs discriminated between patients with abnormal chest radiographs who did or did not have lung cancer, regardless of stage and tumour bulk. The ability of VOCs to detect stage I lung cancer with $100 \%$ sensitivity and $83 \%$ specificity suggests enormous potential as an adjunct to other screening tools. 50

The future: cautious optimism

A NEW ERA FOR LUNG CANCER SCREENING

Screening for lung cancer is apparently entering a new era. After years of frustrating results, new developments $\stackrel{\sigma}{\sigma}$ are being greeted with enthusiasm. Worldwide, groups $\overrightarrow{\overrightarrow{0}}$ have set out to evaluate further the sensitivity of these newer methods. In January 1999 the NCI funded New of Mayo Lung Project was launched to screen for early on lung cancers using low dose helical CT scanning at $\frac{\text { }}{0}$ enrolment and annually for three years thereafter. By December 1500 individuals had been enrolled; they $\rightarrow$ were 50 years of age, current or former smokers (>20 $\mathrm{G}$ pack years), not on supplemental oxygen, and with a life expectancy of more than five years. The aim is to detect $75 \%$ or more of lung cancers at stage I (J R Jett, $\vec{\overrightarrow{ }}$ personal communication). In Florida another pilot study has started using helical CT scanning and sputum $\frac{2}{\omega}$ screening with hnRNP A1/B2 analysis. This study aims $\underset{N}{N}$ to screen 5000 men of high risk and, as lung cancer is 8 more common in those with airflow obstruction 51 all those with a forced expiratory volume in one second $\left(\mathrm{FEV}_{1}\right)$ of $<70 \%$ predicted (about $23 \%$ of those screened) will be enrolled. Subjects will be $>45$ years of age and have smoked $>30$ pack years and the aim is to detect a threefold increase in stage I cancer (M S \& Tockman, personal communication) Other groups in $\overrightarrow{\overrightarrow{0}}$ Germany, Israel, and possibly the UK ${ }^{52}$ are considering establishing their own studies.

SCOPE, COST, AND POTENTIAL BENEFITS

There are many issues that arise from the activity described in this review. Analysis of the data of the 1980s 3 suggests flaws both in study design and interpretation, but there have been substantial developments in tech- $\frac{3}{3}$ nology, therapeutics, and methodology since then. At o present all the effort is focusing on the value of helical CT scanning (together with biological markers in the sputum in some studies) and it is emerging that the incidence of stage I cancers probably will rise to $60 \%$ ᄋ of those identified. However, different groups have $\mathbb{O}$ different algorithms for dealing with NCNs and only $\underset{\omega}{\mathrm{\omega}}$ some are following the Henschke guidelines. Hence, this, together with the inevitable learning curves (the o more multicentred a trial, the more difficult this be- $\mathbb{\Phi}$ comes), will make interpretation less certain.

A more important issue is the selection of the group to screen. Sone et a $l^{21}$ screened a large cohort of relatively $\frac{\vec{O}}{\mathbb{D}}$ young smokers and non-smokers and identified far fewer

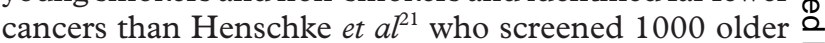
committed smokers. Others are going further by including the presence of airflow obstruction. The choice 8 of population to screen will have a profound effect on cost, and the economic factor is of major concern.

A move to routine screening would represent a fundamental change in our approach to lung cancer. Standard CT scans without contrast cost $£ 500$ at University College London Hospitals. If we assume that there are 12.1 million adult smokers in the UK (and many more 


\section{LEARNING POINTS}

* Mortality in lung cancer is lower for stage I tumours; currently only $20 \%$ of lung cancers are picked up at this stage

* In the 1980s four RCTs failed to show a reduction in lung cancer mortality when male smokers were regularly screened with chest radiography and cytological examination of sputum

* Spiral CT scanning is more sensitive than chest radiography and may pick up $80-85 \%$ of lung cancers at stage I

* Mobile spiral CT scanning picked up 19 lung cancers in 3967 participants in the Matsumoto $\vec{\circ}$ study. Of these, 16 were resectable and $18 \mathrm{had}$ been missed on miniature chest radiography

* ELCAP aims to establish the role of annual low dose spiral CT scanning in screening for peripheral lung cancers. To date, 27 such tumours, of which 26 were resectable, have been identified in 1000 volunteers

* Further mortality and follow up data are required from ELCAP and other sources before national screening programmes can be endorsed

ex-smokers) and that a low dose CT scan costs $£ 150$, the cost of an annual low dose CT scan for every adult smoker will be $£ 1.8$ billion per year, roughly equivalent to $20 \%$ of the government's annual revenue from the tobacco industry and $5 \%$ of the amount spent on tobacco advertising each year. Despite the apparent high cost, the potential benefits and savings of early lung cancer treatment could be enormous. One way to rationalise the programme may be to screen only those at highest risk of lung cancer - that is, those with a long smoking history or an $\mathrm{FEV}_{1}$ of $\leqslant 70 \%$ predicted. Should we also only be screening those who are fit for thoracotomy despite hopes that non-surgical treatments may play a role in early and preinvasive lung cancer? Restriction of screening to high risk groups could reduce the numbers to just over one million but the burden on existing resources would still be heavy. There will be huge demands on scanners, radiologists, chest physicians, and thoracic surgeons which should not be underestimated when such a trial is designed. In addition, automated high throughput detection equipment for sputum cytopathology is being adapted from the Auto-PAP systems used for cervical cancer, making sputum assessment more feasible but, again, at a cost. There is still a lot that we need to know about screening for lung cancer and we need to await the results of the current incidence trials. If these show the anticipated enhanced detection, then a larger international multicentre RCT of helical CT scanning plus biological markers versus conventional chest radiographic screening will need to be performed to ensure that there is an improved cure rate with early detection and resection. The length of lead time bias will be assessable, together with the PPV of screening protocols. The trials should be sufficiently extensive to ascertain the age, smoking history, lung function, and sex for a screening programme. This will require a huge concerted effort and the UK should participate in this venture. Efforts should begin to prepare the way for funding of the appropriate equipment, both CT scanners and automated high throughput cytopathological detection equipment. Let us then hope that such equipment will begin its use in those studies that may eventually change our approach to this disease.
1 Landis SH, Murray T, Bolden S, et al. Cancer statistics. CA Cancer $\mathcal{F}$ Clin 1999;49:8-31.

2 Tong L, Spitz MR, Fueger JJ, et al. Lung carcinoma in former smokers. Cancer 1996;78:1004-10.

3 Midthun DE, Jett JR. Lung tumours. In: Albert RK, Spiro SG, Jett JR, eds. Comprehensive respiratory medicine. London: Harcourt Brace, 1999: 8:43.1-8:43.24.

4 Bignall JR, Martin M, Smithers DW. Survival in 6068 cases of bronchial carcinoma. Lancet 1967; i:1067-70.

5 Edinburgh Lung Cancer Group. Patients presenting with lung cancer in South East Scotland. Thorax 1987;42:853-7.

6 Landis SH, Murray T, Bolden S, et al. Cancer statistics. CA Cancer fُ ڤ้ Clin 1998;48:6-29 (published erratum appears in CA Cancer $\mathcal{F}$ Clin $\stackrel{\mathbb{Q}}{\mathcal{Q}}$ 1998;48:192).

7 Flehinger B, Kimmel M, Melamed M. The effect of surgical treatment $\overline{\bar{o}}$ on survival from early lung cancer. Implications for screening. Chest 1992;101:1013-8.

8 Melamed M, Flehinger B, Zaman M. Impact of early detection on the clinical course of lung cancer. Surg Clin North Am 1987;67:909-24. Nesbitt J, Putnam JJ, Walsh G, et al. Survival in early-stage lung cancer. Ann Thorac Surg 1995;60:466-72.

10 Shah R, Sabanathan S, Richardson J, et al. Results of stage I and II lung cancer. $\mathcal{F}$ Cardiovasc Surg 1996;37:169-72

11 Sobue T, Suzuki R, Matsuda M, et al. Survival for clincal stage I lung cancer not surgically treated. Comparison between screen-detected and symptom-detected cases. The Japanese Lung Cancer Screening Research Group. Cancer 1992;69:685-92.

12 Fontana RS, Sanderson DR, Taylor WF, et al. Early lung cancer detection: results of the initial (prevalence) radiologic and cytologic screening in the Mayo Clinic Study. Am Rev Respir Dis 1984;130: $561-5$

13 Frost JK, Ball WC, Levin ML, et al. Early lung cancer detection: results of the initial (prevalence) radiologic and cytologic screening in the

Johns Hopkins Study. Am Rev Respir Dis 1984;130:549-54.
14 Flehinger BJ, Melamed MR, Zaman MB, et al. Early lung cancer N detection: results of the initial (prevalence) radiologic and cytologic screening in the Memorial Sloan-Kettering Study. Am Rev Respir Dis 1984;130:555-60.

15 Fontana RS, Sanderson DR, Woolner LB, et al. Lung cancer screening: the Mayo program. F Occup Med 1986;28:746-50.

16 Fontana RS, Sanderson DR, Woolner LB, et al. The Mayo Lung Project for early detection and localization of bronchogenic carcinoma: a status report. Chest 1975;67:511-22.

17 Melamed MR, Flehinger BJ, Zaman MB, et al. Screening for early lung \& cancer: results of the Memorial Sloan-Kettering Study in New York. Chest 1984;86:44-53.

18 Tockman M Survival and mortality from lung cancer in a screened $\bar{O}$ population: the Johns Hopkins study. Chest 1986;89(Suppl):324-5S.

9 Kubik A, Parkin DM, Khlat M, et al. Lack of benefit from semi-annual screening for cancer of the lung: follow-up report of a randomized controlled trial of population of high-risk males in Czechoslovakia. Int $\mathcal{F}$ Cancer 1990;45:26-33.

20 Henschke CI, McCauley DI, Yankelevitz DF, et al. Early lung cancer detection project: overall design and baseline screening. Lancet 1999; 354:99-105.

21 Sone S, Takashima S, Li F, et al. Mass screening for lung cancer with mobile spiral computed tomography scanner. Lancet 1998;351: $1242-5$

22 Flehinger BJ, Kimmel M, Polyak T, et al. Screening for lung cancer The Mayo Lung Project revisited. Cancer 1993;72:1573-80.

23 Hammond E, Selikoff I, Seidman H. Asbestos exposure, cigarette smoking and death rates. Ann NY Acad Sci 1979;330:473-90.

Samet J. Radon and lung cancer. F Natl Cancer Inst 1989;81:745-57.

25 Skillrud DM, Offord KP, Miller RD. Higher risk of lung cancer in 
chronic obstructive pulmonary disease. Ann Intern Med 1986;105: $503-7$

26 Sellers T. Familial predisposition to lung cancer. In: Roth J, Cox J, Hong W, eds. Lung cancer. Boston: Blackwell Scientific Publications, 1993: 20-33.

27 Shields P, Harris C. Genetic predisposition to lung cancer. In: Roth J, Cox J, Hong W, eds. Lung cancer. Boston: Blackwell Scientific Publications, 1993: 3-19.

28 Strauss GM. Measuring effectiveness of lung cancer screening. From consensus to controversy and back. Chest 1997;112:216-28S.

29 NCI. http//www.dcpc.nci.nih.gov/PLCO/Default.html 1999.

30 Smart CR. Screening and early cancer detection. Semin Oncol 1990;17: 456-62.

31 Soda $\mathrm{H}$, Tomita $\mathrm{H}$, Kohno $\mathrm{S}$, et al. Limitation of annual screening chest radiography for the diagnosis of lung cancer. A retrospective study. Cancer 1993;15:2341-6.

32 Kaneko M, Eguchi K, Ohmatsu H, et al. Peripheral lung cancer: screening and detection with low-dose spiral CT versus radiography. Radiology 1996;201:798-802.

33 Iinuma T, Tateno Y, Matsumoto T. Comparison of two types of mass screening for lung cancer in terms of cost-effectiveness: indirect chest x-ray vs ISCT. Nippon Igaku Hoshasen Hakkai Sazzhi 1994;54:943-9.

34 Nitta N, Takahashki M, Murata K, et al. Ultra low-dose helicial CT of the chest. AfR 1988;171:383-5.

35 Braithwaite KL, Rabbitts PH. Multi-step evolution of lung cancer. Semin Cancer Biol 1999;9:255-65.

36 Tockman MS, Gupta PK, Myers JD, et al. Sensitive and specific monoclonal antibody recognition of human lung cancer antigen on preserved sputum cells: a new approach to early lung cancer detection. 7 Clin Oncol 1988;6:1685-93.

37 Tockman MS, Erozan YS, Gupta P, et al. The early detection of second primary lung cancers by sputum immunostaining. Lung Cancer Early Detection Group. Chest 1994;106(Suppl):385-90S

38 Qiao Y-L, Tockman MS, Li L, et al. A case-cohort study of an early biomarker of lung cancer in a screening cohort of Yunnan tin miners in China. Cancer Epidemiol Biomarkers Prev 1997;6:893-900.

39 Tockman MS, Mulshine JL, Piantadosi S, et al. Prospective detection of preclinical lung cancer: results from two studies of heterogeneous nuclear ribonucleoprotein $\mathrm{A} 2 / \mathrm{B} 1$ overexpression. Clin Cancer Res 1997;3:2237-46.

40 Fielding P, Turnbull L, Prime W, et al. Heterogenous nuclear ribonucleoprotein A2/B1 upregulation in bronchial lavage specimens: a 0 clinical marker of early lung cancer detection. Clin Cancer Res 1999; 5:4048-52.

41 Mao L, Hruban RH, Boyle JO, et al. Detection of oncogene mutations in sputum precedes diagnosis of lung cancer. Cancer Res 1994;54: $1634-7$.

42 Mulshine JL, Henschke CI. Prospects for lung-cancer screening. Lancet 2000;355:592-3

43 Lam S, MacAulay C, Hung J, et al. Detection of dysplasia and carcinoma in situ by a lung imaging fluorescence endoscope (LIFE) device. $\mathcal{F}$ Thorac Cardiovasc Surg 1993;105:1035-40.

44 Lam S, Kennedy T, Unger M, et al. Localization of bronchial intraepithelial neoplastic lesions by fluorescence bronchoscopy. Chest 1998; 113:696-702.

45 George PJM. Fluorescence bronchoscopy for the early detection of lung $\vec{\circ}$ cancer. Thorax 1999;54:180-3.

46 Scott FM, Modali R, Lehman TA, et al. High frequency of K-ras codon 12 mutations in bronchoalveolar lavage fluid of patients at high risk 12 mutations in bronchoalveolar lavage fluid of patients at high

47 Ahrendt SA, Chow JT, Xu LH, et al. Molecular detection of tumour cells in bronchoalveolar lavage fluid from patients with early stage lung cancer. 7 Natl Cancer Inst 1999:91:332-9.

48 Sozzi G, Musso K, Ratcliffe C, et al. Detection of microsatellite alterations in plasma DNA of non-small cell lung cancer patients. A o prospect for early diagnosis. Clin Cancer Res 1999;5:2689-92.

49 Gazdar AF, Minna JD. Molecular detection of early lung cancer. F Natl $\stackrel{\mathcal{C}}{\mathscr{C}}$ Cancer Inst 1999;91:332-9.

50 Phillips M, Gleeson K, Hughes JMB, et al. Volatile organic compounds 므 in breath as markers of lung cancer: a cross-sectional study. Lancet 1999;353:1930-3.

51 Tockman MS, Anthonisen NR, Wright EC, et al. Airways obstruction and the risk for lung cancer. Ann Intern Med 1987;106:512-8.

52 Dobson R. Screening trial for lung cancer planned for UK. BMf 2000; 